PROCEEDINGS OF THE

AMERICAN MATHEMATICAL SOCIETY

Volume 139, Number 8, August 2011, Pages 2853-2861

S 0002-9939(2011)10833-5

Article electronically published on January 28, 2011

\title{
EXISTENCE OF SOLUTIONS WITH ASYMPTOTIC BEHAVIOR OF EXTERIOR PROBLEMS OF HESSIAN EQUATIONS
}

\author{
LIMEI DAI
}

(Communicated by Matthew J. Gursky)

\begin{abstract}
In this paper, we use the Perron method to prove the existence of viscosity solutions with asymptotic behavior at infinity to Hessian equations.
\end{abstract}

\section{INTRODUCTION}

In this paper, we study the Hessian equation

$$
\sigma_{k}\left(\lambda\left(D^{2} u\right)\right)=1 \text { in } \mathbb{R}^{n} \backslash \partial \Omega,
$$

where $\Omega \subset \mathbb{R}^{n}$ is any strictly convex bounded domain,

$$
\sigma_{k}\left(\lambda\left(D^{2} u\right)\right)=\sum_{i_{1}<\cdots<i_{k}} \lambda_{i_{1}} \cdots \lambda_{i_{k}}, k=1, \cdots, n
$$

is the $k$ th elementary symmetric function of $\lambda\left(D^{2} u\right)=\left(\lambda_{1}, \cdots, \lambda_{n}\right)$, the eigenvalues of the Hessian matrix $D^{2} u$.

The Hessian equation (1.1) is an important class of fully nonlinear elliptic equations. For $k=1$ (1.1) is the Poisson equation $\Delta u=1$, and for $k=n$, (1.1) is the Monge-Ampère equation $\operatorname{det}\left(D^{2} u\right)=1$. There exist many excellent results in bounded domains of Hessian equations; see [3]-[11] and the references therein. For instance, Caffarelli, Nirenberg and Spruck in [3] established the classical solvability of the Dirichlet problem of Hessian equations. Trudinger in 9] demonstrated the existence and uniqueness of weak solutions and Urbas in [11] proved the existence of viscosity solutions.

In 1, Caffarelli and $\mathrm{Li}$ investigated the existence of solutions with asymptotic behavior to Monge-Ampère equations in exterior domains and entire solutions to Monge-Ampère equations. In this paper, we study the solutions with asymptotic behavior of exterior problems of Hessian equations (1.1) using the Perron method.

To work in the realm of elliptic equations, we have to restrict the solutions to the following class of functions. Let

$$
\Gamma_{k}=\left\{\lambda \in \mathbb{R}^{n} \mid \sigma_{j}(\lambda)>0, j=1,2, \cdots, k\right\} .
$$

$\Gamma_{k}$ is a symmetric cone, that is, any permutation of $\lambda$ is in $\Gamma_{k}$ if $\lambda \in \Gamma_{k}$. When $k=1, \Gamma_{k}$ is the half-space $\left\{\lambda \in \mathbb{R}^{n} \mid \sum_{i=1}^{n} \lambda_{i}>0\right\}$. When $k=n, \Gamma_{k}$ is the positive

Received by the editors May 5, 2010 and, in revised form, July 28, 2010.

2010 Mathematics Subject Classification. Primary 35J96, 35J60.

Key words and phrases. Hessian equations, viscosity solutions, asymptotic behavior.

(C)2011 American Mathematical Society 
cone $\Gamma^{+}=\left\{\lambda \in \mathbb{R}^{n} \mid \lambda_{i}>0, i=1, \cdots, n\right\}$. Following [3], we give the definition of $k$-convex functions.

Definition 1.1. A function $u \in C^{2}\left(\mathbb{R}^{n} \backslash \partial \Omega\right)$ is called $k$-convex if $\lambda \in \overline{\Gamma_{k}}$, where $\lambda=\lambda\left(D^{2} u\right)=\left(\lambda_{1}, \lambda_{2}, \cdots, \lambda_{n}\right)$ are the eigenvalues of the Hessian matrix $D^{2} u$.

Definition 1.2. A function $u \in C^{0}\left(\mathbb{R}^{n} \backslash \partial \Omega\right)$ is called a viscosity subsolution of (1.1) if for any $y \in \mathbb{R}^{n} \backslash \partial \Omega, \xi \in C^{2}\left(\mathbb{R}^{n} \backslash \partial \Omega\right)$ satisfying

$$
u(x) \leq \xi(x), x \in \mathbb{R}^{n} \backslash \partial \Omega \quad \text { and } \quad u(y)=\xi(y),
$$

we have

$$
\sigma_{k}\left(\lambda\left(D^{2} \xi(y)\right)\right) \geq 1
$$

A function $u \in C^{0}\left(\mathbb{R}^{n} \backslash \partial \Omega\right)$ is called a viscosity supersolution of (1.1) if for any $y \in \mathbb{R}^{n} \backslash \partial \Omega$ and any $k$-convex function $\xi \in C^{2}\left(\mathbb{R}^{n} \backslash \partial \Omega\right)$ satisfying

$$
u(x) \geq \xi(x), x \in \mathbb{R}^{n} \backslash \partial \Omega \quad \text { and } \quad u(y)=\xi(y),
$$

we have

$$
\sigma_{k}\left(\lambda\left(D^{2} \xi(y)\right)\right) \leq 1
$$

A function $u \in C^{0}\left(\mathbb{R}^{n} \backslash \partial \Omega\right)$ is called a viscosity solution of (1.1) if $u$ is both a viscosity subsolution and a viscosity supersolution of (1.1).

$u$ is a viscosity solution of (1.1) if $u$ is a $k$-convex classical solution of (1.1). Conversely, $u$ is a $k$-convex classical solution of (1.1) if $u$ is a viscosity solution of (1.1) and $u$ is of class $C^{2}$; see [11.

Definition 1.3. A function $u \in C^{0}\left(\mathbb{R}^{n} \backslash \partial \Omega\right)$ is called $k$-convex if in the viscosity sense $\sigma_{j}\left(\lambda\left(D^{2} u\right)\right) \geq 0$ in $\mathbb{R}^{n} \backslash \partial \Omega, j=1,2, \cdots, k$.

$u \in C^{0}\left(\mathbb{R}^{n} \backslash \partial \Omega\right)$ is $k$-convex if and only if $u$ is $C^{0}$ subharmonic; $u$ is $n$-convex if and only if $u$ is convex.

The main result of this paper is the following existence theorem.

Theorem 1.1. Let $k \geq 3$. For any $c \in \mathbb{R}$, there exists a constant $\beta_{0} \in \mathbb{R}$ such that for any $\beta>\beta_{0}$ there exists a $k$-convex viscosity solution $u \in C^{0}\left(\mathbb{R}^{n} \backslash \partial \Omega\right)$ of (1.1) which satisfies

$$
\begin{gathered}
\limsup _{|x| \rightarrow \infty}\left(|x|^{k-2}\left|u(x)-\left[\frac{c_{*}}{2}|x|^{2}+c\right]\right|\right)<\infty, \\
u(x)=-\beta, \quad x \in \partial \Omega,
\end{gathered}
$$

where $c_{*}=\left(\frac{1}{C_{n}^{k}}\right)^{\frac{1}{k}}$ and $\Omega$ is any convex domain in $\mathbb{R}^{n}$.

Remark 1.1. For $k=n$, Theorem 1.1 corresponds to the results in 1], and for entire solution problems of Hessian equations, Theorem 1.1] should also hold.

\section{Preliminaries}

In this section, we prove some lemmas which will be used later.

Lemma 2.1. Let $D$ be a domain in $\mathbb{R}^{n}$ and $f \in C^{0}\left(\mathbb{R}^{n}\right)$ be nonnegative. Assume that the $k$-convex functions $v \in C^{0}(\bar{D}), u \in C^{0}\left(\mathbb{R}^{n}\right)$ satisfy respectively

$$
\begin{gathered}
\sigma_{k}\left(\lambda\left(D^{2} v\right)\right) \geq f(x), x \in D, \\
\sigma_{k}\left(\lambda\left(D^{2} u\right)\right) \geq f(x), x \in \mathbb{R}^{n} .
\end{gathered}
$$


Moreover,

$$
\begin{array}{r}
u \leq v, x \in \bar{D} \\
u=v, x \in \partial D .
\end{array}
$$

Set

$$
w(x)= \begin{cases}v(x), & x \in D, \\ u(x), & x \in \mathbb{R}^{n} \backslash D .\end{cases}
$$

Then $w \in C^{0}\left(\mathbb{R}^{n}\right)$ is a $k$-convex function and satisfies in the viscosity sense

$$
\sigma_{k}\left(\lambda\left(D^{2} w\right)\right) \geq f(x), x \in \mathbb{R}^{n} .
$$

Proof. Let $y \in \mathbb{R}^{n}, \xi \in C^{2}\left(\mathbb{R}^{n}\right)$ satisfy $w(y)=\xi(y)$,

$$
w(x) \leq \xi(x), x \in \mathbb{R}^{n} .
$$

If $y \in D$, we have

$$
v(y)=w(y)=\xi(y), v(x)=w(x) \leq \xi(x), x \in D .
$$

Therefore

$$
\begin{gathered}
\sigma_{j}\left(\lambda\left(D^{2} \xi(y)\right)\right) \geq 0,1 \leq j \leq k, \\
\sigma_{k}\left(\lambda\left(D^{2} \xi(y)\right)\right) \geq f(y) .
\end{gathered}
$$

If $y \in \mathbb{R}^{n} \backslash D$, we have

$$
u(y)=w(y)=\xi(y), u(x)=w(x) \leq \xi(x), x \in \mathbb{R}^{n} \backslash D .
$$

By (2.1), 2.2),

$$
u(x) \leq \xi(x), x \in \mathbb{R}^{n}
$$

Therefore

$$
\begin{gathered}
\sigma_{j}\left(\lambda\left(D^{2} \xi(y)\right)\right) \geq 0,1 \leq j \leq k, \\
\sigma_{k}\left(\lambda\left(D^{2} \xi(y)\right)\right) \geq f(y) .
\end{gathered}
$$

This completes the proof of Lemma 2.1.

The following existence theorem for viscosity solutions on a ball can be found in 4].

Lemma 2.2. Let $B$ be a ball in $\mathbb{R}^{n}$ and $f \in C^{0}(\bar{B})$ be nonnegative. Suppose $\underline{u}, \bar{u} \in C^{0}(\bar{B})$ are respectively the viscosity subsolution and supersolution of

$$
\sigma_{k}\left(\lambda\left(D^{2} u\right)\right)=f(x), x \in B,
$$

and satisfy $\left.\underline{u}\right|_{\partial B}=\left.\bar{u}\right|_{\partial B}=\varphi \in C^{0}(\partial B)$. Then there exists a unique $k$-convex function $u \in C^{0}(\bar{B})$ satisfying

$$
\begin{aligned}
\sigma_{k}\left(\lambda\left(D^{2} u\right)\right) & =f(x), \quad x \in B, \\
u & =\varphi(x), \quad x \in \partial B .
\end{aligned}
$$


Lemma 2.3. Let $B$ be a ball in $\mathbb{R}^{n}$ and $f \in C^{0}(\bar{B})$ be nonnegative. Suppose $\underline{u} \in C^{0}(\bar{B})$ satisfies in the viscosity sense $\sigma_{k}\left(\lambda\left(D^{2} \underline{u}\right)\right) \geq f(x)$ in $B$. Then the Dirichlet problem

$$
\begin{aligned}
\sigma_{k}\left(\lambda\left(D^{2} u\right)\right) & =f(x), \quad x \in B, \\
u & =\underline{u}(x), \quad x \in \partial B
\end{aligned}
$$

has a unique $k$-convex viscosity solution $u \in C^{0}(\bar{B})$.

Proof. Clearly, $\underline{u}$ is a viscosity subsolution of (2.3), (2.4). From Lemma2.2, we only need to prove that (2.3), (2.4) has a viscosity supersolution $\bar{u} \in C^{0}(\bar{B})$ satisfying $\bar{u}=\underline{u}$ on $\partial B$.

Let $v \in C^{2}(B) \cap C^{0}(\bar{B})$ satisfy

$$
\begin{aligned}
\Delta v & =0, \quad x \in B, \\
v & =\underline{u}, \quad x \in \partial B .
\end{aligned}
$$

We claim that $v$ is a viscosity supersolution of (2.3). Indeed, suppose $v$ is not a viscosity supersolution of (2.3). Then there exist $y \in B$ and some $k$-convex function $\xi \in C^{2}(B)$ such that

$$
v(x) \geq \xi(x), x \in B, v(y)=\xi(y),
$$

but

$$
\sigma_{k}\left(\lambda\left(D^{2} \xi(y)\right)\right)>f(y) .
$$

By the $k$-convexity of $\xi$ and the Newton-Maclaurin inequality

$$
\sigma_{1}(\lambda) \geq n\left(\frac{\sigma_{k}(\lambda)}{C_{n}^{k}}\right)^{\frac{1}{k}}, \lambda \in \overline{\Gamma_{k}},
$$

we know that

$$
\Delta \xi(y) \leq n\left(\frac{f(y)}{C_{n}^{k}}\right)^{1 / k}>0
$$

But from (2.5), we get

$$
D^{2} v(y) \geq D^{2} \xi(y)
$$

Hence

$$
\Delta \xi(y) \leq \Delta v(y)=0 .
$$

This is a contradiction. Lemma 2.3 is proved.

The following lemma can be found in [1] or [2].

Lemma 2.4. Let $\Omega \subset \mathbb{R}^{n}$ be a bounded strictly convex domain, $\partial \Omega \in C^{2}, v \in$ $C^{2}(\bar{\Omega})$. Then there exists a constant $c$ only dependent of $n, \Omega,\|v\|_{C^{2}(\Omega)}$ such that for any $\zeta \in \partial \Omega$ there exists $\bar{x}(\zeta) \in \mathbb{R}^{n}$ satisfying

$$
|\bar{x}(\zeta)| \leq c, w_{\zeta}<v, x \in \bar{\Omega} \backslash\{\zeta\},
$$

where

$$
w_{\zeta}(x):=v(\zeta)+\frac{1}{2}\left(|x-\bar{x}(\zeta)|^{2}-|\zeta-\bar{x}(\zeta)|^{2}\right), x \in \mathbb{R}^{n}
$$




\section{Proof of Theorem 1.1}

In this section, we establish the existence theorem of solutions with prescribed asymptotic behavior at infinity to Hessian equations (1.1).

We divide the proof into three steps.

In the first step, we construct a viscosity subsolution of (1.1).

Suppose $\Phi \in C^{\infty}(\bar{\Omega})$ is a $k$-convex function satisfying

$$
\begin{aligned}
\sigma_{k}\left(\lambda\left(D^{2} \Phi\right)\right) & =c_{0}>1, \quad x \in \Omega, \\
\Phi & =0, \quad x \in \partial \Omega .
\end{aligned}
$$

By the comparison principle, $\Phi \leq 0$ in $\Omega$, and by Lemma 2.4, for each $\zeta \in \partial \Omega$, there exists $\bar{x}(\zeta) \in \mathbb{R}^{n}$ such that

$$
w_{\zeta}(x)<\Phi(x), x \in \bar{\Omega} \backslash\{\zeta\},
$$

where

$$
w_{\zeta}(x):=\frac{1}{2}\left(|x-\bar{x}(\zeta)|^{2}-|\zeta-\bar{x}(\zeta)|^{2}\right), x \in \mathbb{R}^{n},
$$

and $\sup _{\zeta \in \partial \Omega}|\bar{x}(\zeta)|<\infty$. Therefore

$$
\begin{gathered}
w_{\zeta}(\zeta)=0, w_{\zeta}(x) \leq \Phi(x) \leq 0, x \in \bar{\Omega} \\
\sigma_{k}\left(\lambda\left(D^{2} w_{\zeta}(x)\right)\right)=C_{n}^{k}>1, x \in \mathbb{R}^{n} .
\end{gathered}
$$

Thus

$$
w(x):=\sup _{\zeta \in \partial \Omega} w_{\zeta}(x), x \in \mathbb{R}^{n}
$$

satisfies

$$
w(x) \leq \Phi(x), x \in \Omega .
$$

From the reference [7, $w$ satisfies

$$
\sigma_{k}\left(\lambda\left(D^{2} w\right)\right) \geq 1, x \in \mathbb{R}^{n} .
$$

Define

$$
V(x)= \begin{cases}\Phi(x), & x \in \Omega, \\ w(x), & x \in \mathbb{R}^{n} \backslash \Omega .\end{cases}
$$

Then $V \in C^{0}\left(\mathbb{R}^{n}\right)$. By (3.1) and Lemma 2.1, $V$ is a $k$-convex function satisfying in the viscosity sense

$$
\sigma_{k}\left(\lambda\left(D^{2} V\right)\right) \geq 1, \quad x \in \mathbb{R}^{n} .
$$

Fix some $R_{1}>0$ such that

$$
\Omega \Subset B_{R_{1}},
$$

and let

$$
R_{2}:=2 R_{1} \sqrt{c_{*}} .
$$

For $a>1$, define

$$
w_{a}(x):=\inf _{B_{R_{1}}} V+\int_{2 R_{2}}^{\mid \sqrt{c_{*} x} x}\left(s^{k}+a\right)^{\frac{1}{k}} d s, x \in \mathbb{R}^{n} .
$$


Then

$$
D^{2} w_{a}=\left(R^{k}+a\right)^{\frac{1}{k}-1}\left(\begin{array}{cccc}
R^{k-1} c_{*} & 0 & \cdots & 0 \\
0 & \left(R^{k-1}+\frac{a}{R}\right) c_{*} & \cdots & 0 \\
\ldots & \ldots & \cdots & \ldots \\
0 & 0 & \cdots & \left(R^{k-1}+\frac{a}{R}\right) c_{*}
\end{array}\right)
$$

where $R=|y|$. So $\lambda\left(D^{2} w_{a}\right) \in \Gamma_{k}, 0<|x|<\infty$, and

$$
\begin{aligned}
& \sigma_{k}\left(\lambda\left(D^{2} w_{a}\right)\right) \\
= & \left(R^{k}+a\right)^{\frac{1}{k}-1}\left(R^{k-1} c_{*} C_{n-1}^{k-1}\left(R^{k-1}+\frac{a}{R}\right)^{k-1} c_{*}^{k-1}+C_{n-1}^{k}\left(R^{k-1}+\frac{a}{R}\right)^{k} c_{*}^{k}\right) \\
= & \frac{1}{C_{n}^{k}}\left(C_{n-1}^{k-1}+C_{n-1}^{k}\left(1+\frac{a}{R^{k}}\right)\right) \\
\geq & \frac{1}{C_{n}^{k}}\left(C_{n-1}^{k-1}+C_{n-1}^{k}\right) \\
= & 1,0<|x|<\infty .
\end{aligned}
$$

By the definition of $R_{2}$,

$$
\left|\sqrt{c_{*}} x\right| \leq \frac{R_{2}}{2},|x| \leq R_{1} .
$$

Consequently

$$
\begin{aligned}
w_{a}(x) & \leq \inf _{B_{R_{1}}} V+\int_{2 R_{2}}^{\frac{R_{2}}{2}}\left(s^{k}+a\right)^{\frac{1}{k}} d s \\
& <\inf _{B_{R_{1}}} V \leq V(x), \quad|x| \leq R_{1} .
\end{aligned}
$$

Fix some $R_{3}>3 R_{2}$ satisfying

$$
R_{3} \sqrt{c_{*}}>3 R_{2}
$$

We choose $a_{1}>1$ such that for $a \geq a_{1}$,

$$
w_{a}(x)>\inf _{B_{R_{1}}} V+\int_{2 R_{2}}^{3 R_{2}}\left(s^{k}+a\right)^{\frac{1}{k}} d s \geq V(x), \quad|x|=R_{3} .
$$

Then by (3.4),

$$
R_{3} \geq R_{1} \text {. }
$$


By the definition of $w_{a}$,

$$
\begin{aligned}
w_{a}(x)= & \inf _{B_{R_{1}}} V+\int_{2 R_{2}}^{\left|\sqrt{c_{*}} x\right|} s\left(\left(1+\frac{a}{s^{k}}\right)^{\frac{1}{k}}-1\right) d s+\int_{2 R_{2}}^{\left|\sqrt{c_{*}} x\right|} s d s \\
= & \inf _{B_{R_{1}}} V+\int_{2 R_{2}}^{\left|\sqrt{c_{*}} x\right|} s\left(\left(1+\frac{a}{s^{k}}\right)^{\frac{1}{k}}-1\right) d s+\frac{c_{*}}{2}|x|^{2}-2 R_{2}^{2} \\
= & \frac{c_{*}}{2}|x|^{2}+c+\inf _{B_{R_{1}}} V+\int_{2 R_{2}}^{\infty} s\left(\left(1+\frac{a}{s^{k}}\right)^{\frac{1}{k}}-1\right) d s-c \\
& -2 R_{2}^{2}-\int_{\left|\sqrt{c_{*}} x\right|}^{\infty} s\left(\left(1+\frac{a}{s^{k}}\right)^{\frac{1}{k}}-1\right) d s, x \in \mathbb{R}^{n} .
\end{aligned}
$$

Let

$$
\mu(a)=\inf _{B_{R_{1}}} V+\int_{2 R_{2}}^{\infty} s\left(\left(1+\frac{a}{s^{k}}\right)^{\frac{1}{k}}-1\right) d s-c-2 R_{2}^{2} .
$$

Then $\mu(a)$ is continuous and monotonic increasing for $a$ and when $a \rightarrow \infty, \mu(a) \rightarrow$ $\infty$. Also,

Moreover,

$$
w_{a}(x)-\mu(a) \leq \frac{c_{*}}{2}|x|^{2}+c, a \geq a_{1}, x \in \mathbb{R}^{n} .
$$

$$
w_{a}(x)=\frac{c_{*}}{2}|x|^{2}+c+\mu(a)+O\left(|x|^{2-k}\right), \text { when }|x| \rightarrow \infty .
$$

For $a \geq a_{1}$, set $\beta_{0}=\mu(a)$ and define, for any $\beta>\beta_{0}$,

$$
\underline{u}_{a}(x)= \begin{cases}\max \left\{V(x), w_{a}(x)\right\}-\beta, & |x| \leq R_{3}, \\ w_{a}(x)-\beta, & |x| \geq R_{3} .\end{cases}
$$

Then

$$
\underline{u}_{a}(x)=-\beta, x \in \partial \Omega,
$$

and by (3.6),

$$
\underline{u}_{a}(x) \leq \frac{c_{*}}{2}|x|^{2}+c+O\left(|x|^{2-k}\right), \text { when }|x| \rightarrow \infty .
$$

Choose $a_{2} \geq a_{1}$ sufficiently large such that for $a \geq a_{2}$,

$$
\begin{aligned}
V(x)-\beta & \leq V(x)-\beta_{0} \\
& =V(x)-\inf _{B_{R_{1}}} V-\int_{2 R_{2}}^{\infty} s\left(\left(1+\frac{a}{s^{n}}\right)^{\frac{1}{k}}-1\right) d s+c+2 R_{2}^{2} \\
& \leq c \leq \frac{c_{*}}{2}|x|^{2}+c, \quad|x| \leq R_{3} .
\end{aligned}
$$

Therefore

$$
\underline{u}_{a}(x) \leq \frac{c_{*}}{2}|x|^{2}+c, a \geq a_{2}, x \in \mathbb{R}^{n} .
$$

By Lemma 2.1, $\underline{u}_{a} \in C^{0}\left(\mathbb{R}^{n}\right)$ is $k$-convex and satisfies in the viscosity sense

$$
\sigma_{k}\left(\lambda\left(D^{2} \underline{u}_{a}\right)\right) \geq 1, \quad x \in \mathbb{R}^{n} .
$$


In the second step, we define the Perron solution of (1.1).

For $a \geq a_{2}$, let $\mathbb{S}_{a}$ denote the set of $k$-convex functions $v \in C^{0}\left(\mathbb{R}^{n}\right)$ satisfying

$$
\begin{gathered}
\sigma_{k}\left(\lambda\left(D^{2} v\right)\right) \geq 1, \quad x \in \mathbb{R}^{n} \backslash \partial \Omega, \\
v(x)=-\beta, \quad x \in \partial \Omega, \\
v(x) \leq \frac{c_{*}}{2}|x|^{2}+c, \quad x \in \mathbb{R}^{n} .
\end{gathered}
$$

Clearly, $\underline{u}_{a} \in \mathbb{S}_{a}$. Hence $\mathbb{S}_{a} \neq \emptyset$. Define in $\mathbb{R}^{n}$,

$$
u_{a}(x):=\sup \left\{v(x) \mid v \in \mathbb{S}_{a}\right\}
$$

Then $u_{a}$ is a viscosity subsolution of (1.1) and satisfies

$$
\begin{gathered}
u_{a}(x)=-\beta, \quad x \in \partial \Omega, \\
u_{a}(x) \leq \frac{c_{*}}{2}|x|^{2}+c, \quad x \in \mathbb{R}^{n} .
\end{gathered}
$$

In the following, we prove that $u_{a}$ is a viscosity supersolution of (1.1).

In the third step, we prove that $u_{a}$ is a viscosity solution of (1.1).

For any $x_{0} \in \mathbb{R}^{n} \backslash \partial \Omega$, choose a ball $B=B_{\varepsilon}\left(x_{0}\right) \subset \mathbb{R}^{n} \backslash \partial \Omega$. By Lemma 2.3. the Dirichlet problem

$$
\begin{aligned}
\sigma_{k}\left(\lambda\left(D^{2} \tilde{u}\right)\right) & =1, \quad x \in B, \\
\tilde{u} & =u_{a}, \quad x \in \partial B
\end{aligned}
$$

has a $k$-convex viscosity solution $\tilde{u} \in C^{0}(\bar{B})$. From the comparison principle,

$$
u_{a} \leq \tilde{u}, x \in B \text {. }
$$

Define

$$
\psi(x)= \begin{cases}\tilde{u}(x), & x \in B \\ u_{a}(x), & x \in \mathbb{R}^{n} \backslash(B \cup \partial \Omega) .\end{cases}
$$

By Lemma 2.1.

$$
\sigma_{k}\left(\lambda\left(D^{2} \psi(x)\right)\right) \geq 1, x \in \mathbb{R}^{n} \backslash \partial \Omega .
$$

Because

$$
\begin{aligned}
\sigma_{k}\left(\lambda\left(D^{2} \tilde{u}\right)\right) & =1=\sigma_{k}\left(\lambda\left(D^{2} g\right)\right), & & x \in B, \\
\tilde{u} & =u_{a} \leq g, & & x \in \partial B,
\end{aligned}
$$

where $g(x)=\frac{c_{*}}{2}|x|^{2}+c$, from the comparison principle,

$$
\tilde{u} \leq g, \quad x \in B .
$$

Hence $\psi \in \mathbb{S}_{a}$.

By the definition of $u_{a}, u_{a} \geq \psi$ in $\mathbb{R}^{n}$. Consequently $\tilde{u} \leq u_{a}$ in $B$. As a result,

$$
\tilde{u}=u_{a}, \quad x \in B \text {. }
$$

Because $x_{0}$ is arbitrary, we know that $u_{a}$ is a viscosity supersolution of (1.1).

By the definition of $u_{a}$,

$$
\underline{u}_{a} \leq u_{a} \leq g, x \in \mathbb{R}^{n},
$$

so $u_{a}$ satisfies (1.2). Theorem 1.1 is proved. 


\section{ACKNOWLEDGEMENTS}

The author would like to thank Professor Jiguang Bao for his guidance and Professor Luis Caffarelli and Professor Yanyan Li for their suggestions. The author is also indebted to the referee for the deep and helpful comments.

\section{REFERENCES}

[1] L. Caffarelli, Y. Y. Li, An extension to a theorem of Jörgens, Calabi, and Pogorelov, Comm. Pure Appl. Math. 56 (2003) 549-583. MR1953651 (2004c:35116)

[2] L. Caffarelli, Y. Y. Li, Some multi-valued solutions to Monge-Ampère equations, Comm. Anal. Geom. 14 (2006) 411-441. MR2260718 (2007i:35067)

[3] L. Caffarelli, L. Nirenberg, J. Spruck, The Dirichlet problem for nonlinear second-order elliptic equations. III. Functions of the eigenvalues of the Hessian, Acta Math. 155 (1985) 261-301. MR806416 (87f:35098)

[4] A. Colesanti, P. Salani, Hessian equations in non-smooth domains, Nonlinear Anal. 38 (1999) 803-812. MR 1710156 (2001i:35088)

[5] K. S. Chou, X. J. Wang, A variational theory of the Hessian equation, Comm. Pure Appl. Math. 54 (2001) 1029-1064. MR.1835381 (2002e:35072)

[6] B. Guan, The Dirichlet problem for a class of fully nonlinear elliptic equations, Comm. Partial Differential Equations 19 (1994) 399-416. MR1265805 (95c:35100)

[7] H. Ishii, P. L. Lions, Viscosity solutions of fully nonlinear second-order elliptic partial differential equations, J. Differential Equations 83 (1990) 26-78. MR1031377 (90m:35015)

[8] N. S. Trudinger, On the Dirichlet problem for Hessian equations, Acta Math. 175 (1995) 151-164. MR1368245 (96m:35113)

[9] N. S. Trudinger, Weak solutions of Hessian equations (English summary), Comm. Partial Differential Equations 22 (1997) 1251-1261. MR.1466315 (99a:35077)

[10] N. S. Trudinger, X. J. Wang, Hessian measures. II, Ann. of Math. (2) 150 (1999) 579-604. MR $1726702(2001 f: 35141)$

[11] J. I. E. Urbas, On the existence of nonclassical solutions for two classes of fully nonlinear elliptic equations, Indiana Univ. Math. J. 39 (1990) 355-382. MR1089043 (92h:35074)

School of Mathematics and Information Science, Weifang University, Shandong 261061, People's Republic of China

E-mail address: limeidai@yahoo.com.cn 